\title{
El concepto de Derecho Mayor: una aproximación desde la cosmología andina
}

Carlos Albeiro Flórez-Vargas*

\footnotetext{
Magíster en Educación Ambiental. Profesor, Universidad Libre, sede Cali, Colombia.

Correo electrónico:

primerodignidad@hotmail.com
}

Recibido: 11 de noviembre del 2015

Aprobado: 22 de mayo del 2016

Cómo citar este artículo: Carlos Albeiro Flórez-Vargas. El concepto de Derecho Mayor: una aproximación desde la cosmología andina. DIXI 24. Octubre 2016. Pág. 63. doi: http://dx.doi.org/10.16925/di.v18i24.1523

\section{Resumen}

Introducción: el artículo aborda el concepto de Derecho mayor, el cual ha tomado fuerza en las investigaciones académicas. Dicho concepto se encuentra envuelto en una complejidad estimulante para los investigadores, lo cual invita a la reflexión sobre la mejor forma de comprender los conceptos cosmo-ancestrales y de ordenamiento de la comunidad misak. Metodología: la investigación que da origen a este artículo es de tipo IAP (Investigación Acción Participativa), en la cual la comunidad misak tiene una participación directa en la producción de conocimientos concretos sobre su propia realidad. Resultados: la investigación arroja como resultado la generación de nuevo conocimiento sobre la concepción indígena de Derecho mayor, la cual se basa en la armonía y los principios de correspondencia y complementariedad. Conclusión: el artículo permite comprender cuestiones básicas del pensamiento misak desde el Derecho mayor, haciendo uso de los principios rectores de la cosmología andina a partir de un retrato cosmogónico de la comunidad misak.

Palabras clave: comunidad indígena, cosmología andina, cultura occidental, derecho mayor. 


\title{
The Concept of Greater Right: An Approach from Andean Cosmology
}

\begin{abstract}
Introduction: This article discusses the concept of greater right, which has taken hold in academic research. Steeped in complexity that proves intriguing for researchers, this concept invites reflection on the best way to understand the ancestral worldview and ordering concepts of reality of the Misak community. Methodology: The research that culminated in this article was participatory action research (PAR), in which the Misak community had direct participation in the production of concrete knowledge about their reality. Results: The research yielded the generation of new knowledge about the indigenous conception of greater right, which is based on harmony and the principles of correspondence and complementarity. Conclusion: The article provides insight into basic issues of Misak thought through understanding of greater right, utilizing the guiding principles of Andean cosmology to create a cosmological portrait of the Misak community.
\end{abstract}

Keywords: indigenous community, Andean cosmology, Western culture, greater right.

\section{O conceito de Lei Maior: uma aproximação a partir da cosmologia andina}

\section{Resumo}

Introdução: o artigo aborda o conceito de Lei maior, a qual tem tomado força nas pesquisas acadêmicas. Esse conceito encontra-se envolvido numa complexidade estimulante para os pesquisadores, o qual convida a refletir sobre a melhor forma de compreender os conceitos cosmo-ancestrais e de ordenamento da comunidade misak. Metodologia: a pesquisa que dá origem a este artigo é de tipo IAP (Investigação Ação Participativa) na qual a comunidade misak tem uma participação direta na produção de conhecimentos concretos sobre sua própria realidade. Resultados: a pesquisa mostra como resultado a geração de novo conhecimento sobre a concepção indígena de Lei maior, a qual se baseia na harmonia e nos princípios de correspondência e complementariedade. Conclusão: o artigo permite compreender questões básicas do pensamento misak a partir da Lei maior, fazendo uso dos princípios orientadores da cosmologia andina a partir de um retrato cosmogônico da comunidade misak.

Palavras-chave: comunidade indígena, cosmologia andina, cultura ocidental, Lei maior. 


\section{INTRODUCCIÓN}

El presente artículo tiene como objetivo fundamental presentar una serie de ideas y argumentos razonables que permitan realizar una aproximación a la concepción del Derecho mayor, tomando como punto de partida la cosmología andina, en particular la del pueblo misak. A fin de cumplir con el objetivo central mencionado, se han desarrollado una serie de subtemas - en total siete-, que serán abordados a lo largo del artículo.

Los primeros cuatro asuntos que se desarrollarán están vinculados, de manera estrecha, con algunas de las cuestiones más resonadas en el campo de la cosmología. El quinto tema de análisis está relacionado con el papel del indígena en el cosmos, y los dos últimos con la noción de Derecho mayor y las expectativas que genera dicho término en la actualidad.

En este orden de ideas, es preciso aquí señalar que la cosmología occidental puede presentar sus interrogantes de una manera que se antoja distante de la cosmología desarrollada por las comunidades andinas por cientos de años. No obstante, en este artículo se desarrollan algunas ideas expuestas por Estermann ${ }^{1}$, quien ha elaborado una serie de investigaciones - a nivel cosmológico y filosófico- de las enseñanzas que tiene para brindar el mundo indígena, el mundo andino o, en sus palabras, la "filosofía andina", al mundo occidental. La obra de Estermann que aquí se utilizará como sustento teórico permite establecer un diálogo entre la cosmología indígena y la cosmología occidental. Este diálogo abre la posibilidad de traducir el significado del Derecho mayor a un lenguaje occidental. Para lograr dicha traducción es preciso que se analicen ciertas nociones que, en síntesis, ubican al misak en una posición frente al cosmos y permiten comprender el significado de Derecho Mayor.

Estermann considera que traducir una cosmología indígena al lenguaje occidental no es una tarea fácil. No obstante, postula que es posible realizar tal labor si se tienen en cuenta ciertos principios lógicos que ha detectado mediante años de investigación. Así, de acuerdo con este autor, para "tener una idea más clara de la cosmovisión andina, tenemos que tomar en cuenta siempre los principios lógicos de la racionalidad andina, en especial los principios de correspondencia y complementariedad" ${ }^{2}$. El principio de

1 Cf. Josef Estermann. Filosofía ANDINA: SABIDURÍA INDÍGeNa PARA UN MUNDo NUEvo. Instituto Superior Ecuménico Andino de Teología. (2006).

2 Id. Pág. 158. correspondencia hace alusión a la forma en que los distintos elementos, "aspectos, regiones o campos de la realidad se corresponden de una manera armonio$\mathrm{sa}^{\prime \prime}$. Se trata de un principio que indica la relación necesaria que hay entre los elementos del universo, no de una manera causal, como se acostumbra en el pensamiento occidental, sino de una manera relacional, mutua y bidireccional: es una especie de isomorfismo cósmico que demuestra que lo macro se refleja en lo micro.

En cuanto al principio lógico de complementariedad, se puede decir que es útil en la interpretación de la cosmología andina, toda vez que sobre este se explica la relación especial que existe entre algunos elementos. Estermann explica que no existe algún elemento, o acción alguna, que no co-exista, de manera especial, "con su complemento específico. Este complemento es el elemento que recién hace pleno o completo al elemento correspondiente"4.

Ahora bien, de lo anterior se puede colegir que es preciso, si se quiere elaborar una aproximación a la noción de Derecho mayor a partir de un análisis de la cosmología, tener en cuenta que los argumentos deben construirse tomando como referencia los principios de correspondencia y complementariedad. De ahí que este artículo fundamente sus afirmaciones e ideas sobre los principios mencionados.

Es importante además resaltar, antes de iniciar el recorrido por los tópicos anunciados, que gran parte de la información que sirvió como material de análisis surge del registro de las intervenciones presenciadas en el Primer Encuentro de Autoridades Ancestrales sobre Derecho Mayor. La información recabada en el mencionado encuentro permite que la aproximación aquí exhibida tenga un contenido empírico importante y, de esta manera, se facilite el proceso de análisis y comprensión de los conceptos claves.

\section{LA EXISTENCIA DEL MISAK EN EL COSMOS}

Una primera cuestión importante para ubicar el papel del misak en el cosmos y el significado del Derecho mayor es la que se relaciona con la existencia, con la vida humana. Preguntarse por lo que significa vivir es una costumbre extendida y considerada crucial en muchas culturas occidentales. Algunas respuestas

Id. Pág. 136

Id. Pág. 139. 
filosóficas se encaminan a resolver esta pregunta por medio de la biología: vivir es tener signos vitales. Otras respuestas de este corte se inclinan por una respuesta metafísica: vivir es pensar. Sea cual fuera la respuesta que se localice en el pensamiento occidental, esta se encontrará por fuera de las dimensiones existenciales de los misak.

Para los misak es importante recalcar que la vida se trata de una serie de actividades. Estar vivo es estar actuando, como bien lo señala el rector de la Misak Universidad en el marco del Primer Encuentro de Autoridades Ancestrales sobre Derecho MayorMisak Universidad: "La vida es un conjunto de actividades que hay que desarrollar" 5 . Ahora bien, nótese que no se trata solo de realizar actividades, sino también de actividades que son de carácter obligatorio. El misak nace con una obligación: la de hacer, de realizar ciertas tareas. No se trata de un individuo arrojado libre al mundo, es un sujeto que debe cumplir con unas obligaciones precisas, no solo frente a la comunidad, sino frente a la vida. Incluso, como bien lo hace Peña (2009), se puede afirmar que no existe forma de comprender la vida sin la actividad, sin el hacer: "No existen palabras para expresar el hecho de vivir, por un lado, y de hacer, por el otro; se vive haciendo algo en alguna posición: sentado, parado, colgado, acostado o andando"6.

La obligatoriedad de las actividades de los misak, de aquellas labores para las que han nacido, tiene su sustento en el carácter atemporal de sus relatos cosmogónicos. Para los misak el tiempo no significa lo mismo que para las culturas occidentales. Según Peña (2009):

El tiempo tiene que ver con un modo de hacer en particular y, en esa medida, con un modo de ser desde el que se define un carácter, se fijan aspiraciones, nociones de bienestar y planes de vida; el tiempo tiene que ver con la forma como se disponen las gentes de un pueblo para asumir la existencia ${ }^{7}$.

Es decir, para los misak el tiempo no es algo que, de manera inexorable, transcurre independientemente de las actividades que realice la persona. En el pensamiento misak el tiempo no es algo medible ni que pueda desvincularse con el hacer: "el tiempo

Tata Floro Tunubala. Primer Encuentro de Autoridades Ancestrales sobre Derecho Mayor-Misak Universidad. Valle de Wuampia. Abril 20 del 2015.

6 Véase Joana Peña. En las voces del pishimisak; apuntes etnográficos sobre el tiempo misak. MAguARÉ 23. 2009. Pág. 364.

7 Id. Pág. 363. existe cuando alguien hace algo y no al modo moderno en cuanto condición externa e independiente de los sujetos conforme el supuesto kantiano; es alguien haciendo, siendo; el tiempo se vive como algo interno para el misak". El tiempo está al interior de cada sujeto y transcurre en la medida en que se actúa. Cabe anotar aquí que en "lengua Namuy Wam no hay un término equivalente al de tiempo"

\section{CoSMOgoníA Y GÉNERO}

El origen del mundo, segundo tema importante para elaborar una comprensión cosmológica del Derecho mayor, se encuentra relacionado con el carácter atemporal del pensamiento misak. Los misak son creaturas, son existencias creadas por un ser supremo. Ahora bien, este ser supremo tiene una calidad inmanente. Según Estermann, "lo divino es parte integral del universo o de la realidad (pacha) y no le es trascendente."10. Es decir, el panteísmo se presenta como un rasgo fundamental de la cosmovisión de los misak. La divinidad de estas comunidades se encuentra presente en cada actividad que realiza la naturaleza. Sus expresiones no se encuentran reveladas en algún tipo de escritura santa o revelación divina. Para los misak existen diferentes formas de transmitir las ideas diferentes a la escritura.

En los distintos fenómenos que acontecen a diario los misak pueden observar no solo la obra de su creador, sino a su creador mismo. Este creador inmanente se revela, de manera clara, a través de la tierra y de todo lo que en ella acontece. Como bien lo señala Estermann, "el cosmos (pacha) es cuatrodimensional, una red interconectada de relaciones espacio-temporales. Por eso, el tiempo andino está estrechamente ligado a los fenómenos pachasóficos de tipo astronómico y ecosófico"11. La tierra, la pacha, da cuenta de la existencia del tiempo solo en cuanto algo está ocurriendo. De ahí que cada evento, o cada fenómeno natural, posea cierta carga de sabiduría. Hay sabiduría, pachasofía, en la lluvia, en el resplandor del sol, en el correr de los ríos, en el cantar de las aves, etc.

Una característica importante para la comprensión de la naturaleza de esta divinidad que creó a la comunidad misak es la necesidad de orden y equilibrio

\footnotetext{
Id. Pág. 365.

Id. Pág. 365.

10 Véase Josef Estermann, supra, nota 1. Pag.164.

11 Id. Pág. 196.
} 
para el sano desarrollo del cosmos. Es preciso que las relaciones entre los individuos y la naturaleza sean armónicos, de tal manera que se mantenga la "salud" de la comunidad. La relación con el creador se ve afectada, enferma, en la medida en la que se desequilibre la interdependencia entre los misak y su entorno. En palabras de Estermann:

Inclusive el cosmos entero, la naturaleza del medio ambiente o la religiosidad pueden estar sanos o enfermos. Los parámetros universales podemos llamar equilibrio cósmico (para salud) o desequilibrio cósmico (para enfermedad); cada ruptura de las relaciones vitales es causa o motivo para las enfermedades ${ }^{12}$.

En otras palabras, el universo entero se ve afectado por la desarmonía que pueda darse entre los misak y el entorno, entre los sujetos y la naturaleza, entre los individuos y su creador.

Cabe abordar en este punto el tema relacionado con la forma en la que está ordenado el cosmos, la manera en la que las cosas existentes se relacionan entre sí. Para esto es necesario tener en cuenta que en la cosmología andina la interrelación de los géneros da cuenta del origen y el equilibrio que debe mantenerse. Es decir, en la división entre hombres y mujeres se ve reflejada, para la pachasofía misak, las condiciones de correspondencia presentes en el origen y en el equilibrio que estas deben mantener.

El origen de los sujetos está muy signado en las cosmologías andinas por la representación genérica de los elementos de la naturaleza. La luna representa la mujer, y el sol al hombre, por ejemplo. En el origen se encuentra la división de géneros, mas no se halla caracterizada por la diferenciación de los actores, sino por la capacidad de estos para complementarse. Es decir, la división entre hombres y mujeres se usa en la cosmogonía misak como un modelo ordenado de interacción que explica el origen.

La relación complementaria entre los géneros masculino y femenino, en la cosmología misak, se puede observar como un monumento al cosmos, al orden que se debe mantener. De ahí que sexualidad sea una expresión clara del funcionamiento del universo. Estermann, para referirse a una diferencia fundamental en la forma de abordar la sexualidad entre la filosofía occidental y la pachasofía, afirma: "la filosofía andina, en contraste (...) con la filosofía occidental, (...) interpreta la condición sexual como una constitución universal y hasta cósmica: todo es sexuado en cierto sentido, desde los entes inorgánicos

12 Id. Pág. 236 hasta los fenómenos meteorológicos, cósmicos y religiosos"13. De esta manera, la cosmología misak abarca la existencia del hombre y la mujer integrándola a la existencia de los fenómenos naturales y, por supuesto, del creador. El hombre y la mujer existen como complemento en la medida en la que hacen parte del mundo y en este se refleja dicha relación. Como bien lo explica Estermann:

Cielo y tierra, sol y luna, claro y oscuro, verdad y falsedad, día y noche, bien y mal, masculino y femenino (...) para la cosmología andina (...) no son contraposiciones excluyentes, sino complementos necesarios para la afirmación de una entidad superior e integral ${ }^{14}$.

\section{ComunidAd y Cosmología}

Vivir es hacer, como se señaló con anterioridad. Por lo tanto, lo que se hace, las labores, son importantes para los misak. Dentro de su cosmología, sus labores se encuentran bien definidas en lo relativo al papel de los hombres y las mujeres en la comunidad. En algunos aspectos hay una división sexual del trabajo muy marcada:

La mujer está a cargo de lo relacionado con la preparación de alimentos, el cuidado de los niños, el tejido de prendas, las actividades del hogar; pero en lo concerniente al trabajo de la tierra - cosechar, cultivar, paliar, arar-mujeres y hombres trabajan igual ${ }^{15}$.

Ahora bien, se puede observar que la mujer tiene obligaciones y trabajos que solo ella debe desarrollar, adicional a los trabajos que realiza junto a los hombres. Esto hace de la mujer una figura central en la cosmología misak. La mujer representa un elemento fundamental en la compresión de la comunidad dentro de la cosmología.

Las mujeres representan la tierra, según la cosmovisión andina. La tierra y la mujer proveen la vida, por ello son símbolo de fertilidad. Cuando las semillas de la comunidad y la tierra están en armonía, es decir, saludables, es posible que se geste la vida y la comunidad prospere. Solo la armonía genera prosperidad y crecimiento; los actos que provienen de la enfermedad (desarmonización) no permiten que la comunidad se desarrolle.

\footnotetext{
13 Id. Págs. 223-224.

14 Id. Pág. 142.

15 Véase Joana Peña, supra, nota 7. Pág. 134.
} 
El cuidado de las mujeres y de la tierra se configura como el propósito fundamental de los ritos desplegados por los miembros de la comunidad. Estos ritos, además de perseguir el mencionado objetivo, integran en un proceso práctico la celebración de la armonía del sujeto con la naturaleza y con su creador. Esto se refleja claramente en las palabras de Estermann: "Para la filosofía andina, más se trata de una mediación celebrativa, es decir: las posiciones complementarias llegan realmente a complementarse (integrarse), a través del ritual celebrativo, mediante un proceso pragmático (acción) de integración simbólica"16. Lo anterior indica como los rituales, que están diseñados para cuidar de la tierra y de la fertilidad de la comunidad, reestablecen simbólicamente el cosmos integrando los principios de complementariedad y correspondencia.

El principio de correspondencia se integra en la comunidad a través de los ritos o meditación celebrativa, cuando se muestra que los individuos y su relación entre si no pueden desligarse del entorno, del medio ambiente. En los ritos se rememora la importancia de la tierra y de la fertilidad de las mujeres para el funcionamiento de las demás áreas o aspectos de la comunidad: si hay fertilidad, tanto en la tierra como en las mujeres, hay armonía y los ritos son una expresión acabada de esto.

El principio de complementariedad se refleja en los rituales por medio de la asignación de un rol a cada miembro en estos. Es decir, cuando los rituales contienen una división de los roles y de tareas que cada uno de los participantes debe desempeñar, lo que se busca reforzar es la idea de unidad, de cooperación, de hermandad. La complementariedad está por doquier en los rituales que contienen animales, por ejemplo. La comunidad necesita de ellos y ellos necesitan de la comunidad. Para el misak, los animales son sus hermanos.

Es importante aportar al respecto la perspectiva de un autor familiarizado con los rituales de la región habitada por los misak. En ese sentido, Vasco tiene una visión interesante - la cual bien puede entenderse por medio de los acontecimientos actuales-, acerca de la importancia de los rituales, en especial de las mingas:

Las mingas continúan siendo una práctica muy generalizada entre los guambianos, aunque con mucha menor frecuencia y dimensión de lo que se hacía; su realización está más relacionada con la adquisición y consolidación de un prestigio comunitario, con el

16 Véase Josef Estermann, supra, nota 1. Pág.142. refuerzo de los lazos familiares y veredales, y sobre todo con la revitalización de un sentimiento comunitario y étnico ${ }^{17}$.

Con la frase anterior, se comprende aún más la importancia de la tradición oral y ritual de la cosmogonía para el desarrollo de la comunidad por medio de la celebración de ritos. Sin embargo, se hace preciso aquí, al tratarse de tradición oral, que el argumento, o mejor, la idea sea reforzada por las declaraciones de un miembro de la comunidad:

Las cosmovisiones que... pues... vienen, gracias a nuestros mayores yo digo que no lo hay escrito, sí hay formas de escribir, pero siempre lo hemos tenido en la oralidad, gracias a eso hemos podido los quinientos años más ${ }^{18}$.

El rol de cada individuo dentro de la sociedad viene dado desde el nacimiento. Se trata de una cosmovisión que permite comprender la necesidad de ciertas tareas y lo menesteroso de trabajar con un sentido comunitario.

\section{LA COMUNIDAD MISAK Y LA COMUNIDAD OCCIDENTAL}

Obtener y procesar información acerca de la cosmovisión de una comunidad indígena no es suficiente para comprender su universo. Hace falta establecer un diálogo casi simbiótico que permita la asimilación de símbolos. Esto hace que las relaciones entre las comunidades indígenas y las culturas occidentales, de cara a cumplir con el mandato constitucional de fortalecer la jurisdicción especial indígena, deban ser más cercanas. Ahora bien, ¿hasta dónde estas relaciones pueden ser armónicas sin que una cultura absorba a otra? Dar una respuesta a esta pregunta podría convertirse en un despropósito; sin embargo, es una cuestión que estimula la imaginación de quienes buscan comprender la cosmología de los misak.

Las relaciones entre la comunidad misak y los ciudadanos de cultura occidental no han sido del todo cordiales y armoniosas. Según miembros de la comunidad, "después de mi 1991, que es el reconocimiento

\footnotetext{
17 Véase Luis Vasco. Conceptos Básicos de la Cosmovisión Guambiana en relación con sus procesos de lucha. ReLATORÍA. 1997. Pág. 5. Disponible en: http://www.luguiva.net/articulos/ detalle. aspx?id=38

18 Tata Floro Tunubala. supra, nota 6.
} 
de los derechos, hace ya veinte y dos años, que es un tiempo muy pequeño para la vida de los pueblos indígenas: ¿qué ha pasado de 1991 a 2015 ?"19. La pregunta genera cierta inquietud en quien la lee y sabe de donde proviene. Lo cierto es que se puede percibir cierto dejo de frustración que permite entrever que, tal vez, no han recibido lo que esperaban.

Los esfuerzos por mejorar las relaciones entre la comunidad misak y las otras comunidades, indígenas y no-indígenas, han sido notorios. La Misak Universidad tiene el objetivo trascendental o meta crucial de posibilitar la compresión de las diversas cosmologías con las que debe enfrentarse un miembro de la comunidad. De ahí que su rector se haya expresado con las palabras que siguen:

La educación que había que implementar a través de la cosmovisión, a través de los usos y costumbres y que la escuela tenía que ser una escuela distinta a las demás escuelas, distinta no en infraestructura sino en su contenido curricular, que había que conocer los dos mundos, el mundo propio y el mundo occidental, que había que buscar"20.

No obstante, el sentimiento de muchos miembros de la comunidad apunta hacia un hermetismo, hacia un caminar lejos de las sendas de la cultura occidental. Es la tierra y el derecho que tienen sobre ella los misak de manera ancestral lo que ha producido la mayor cantidad de desacuerdos y de desarmonización entre esta comunidad y el hombre occidental. Pero es también el derecho a la tierra, a la fertilidad, a la prosperidad de la comunidad lo que posibilita que en la actualidad se logre un diálogo franco entre las partes. Es la tierra la que hace el llamado a la tregua, la que ha convocado a las autoridades ancestrales para hacer notar que el Derecho mayor está vivo.

\section{Aproximación al Derecho mayor}

No es posible conocer el significado del Derecho mayor sin antes haber trasegado por la cosmología de la comunidad, y haber comprendido la importancia que tiene la mujer y la tierra para sus miembros. Todo en la cosmogonía habla de la complementariedad entre el hombre y la mujer; de la correspondencia

19 Mama Liliana Pechen Muelas. Primer Encuentro de Autoridades Ancestrales sobre Derecho Mayor-Misak Universidad. Valle de Wuampia. Abril 20 del 2015.

20 Tata Floro Tunubala. Tata Floro Tunubala. supra, nota 6. de todas las áreas de la vida con la tierra, la mujer y la naturaleza. De ahí que sea preciso aproximarse a la noción central de este artículo haciendo uso de las palabras pronunciadas por los asistentes al Primer Encuentro de Autoridades Ancestrales sobre Derecho Mayor, y al tomar como referencia la simbología relacionada en las líneas anteriores.

El Derecho mayor es un derecho directamente asociado con la tierra, con la herencia de los ancestros que poblaron ciertos territorios hace cientos de años. Por lo tanto, es importante describir esta noción teniendo en cuenta que se trata, en esencia, de un derecho al territorio.

Cabe mencionar que el "territorio guambiano es un territorio que está organizado teniendo como ejes los flujos del agua y también con la visión de un territorio sexuado" 21 . Se trata de un territorio que simboliza la complementariedad cuando se representa como si este tuviese "una parte femenina del territorio a la izquierda del río Piendamó y una parte masculina a su derecha"22. Cuando los terratenientes invadieron las zonas pobladas por los misak, solo se apoderaron de "lo fundamental de la parte femenina. Todo el proceso de lucha, entonces, se orientó a recuperar esa parte de su territorio, para volver a reconstituir el par"23. Lo anterior es comprensible, dada la necesidad cosmológica de la comunidad por mantener el principio de complementariedad que fundamenta su lógica particular.

Ahora bien, como se trata de Derecho mayor, de derecho a la tierra, de derecho ancestral, las normas que contiene dicho Derecho deben ser leyes mayores o leyes de origen. Se trata de leyes que han de ser dictadas por un ser superior, por el creador. Esto en razón a que este creador no trasciende su obra, sino que es inmanente a ella, que no es un ser que se encuentre por fuera del planeta, por fuera de su obra. Al tratarse de un ser que convive con su creación y que no se expresa por medio de escrituras reveladas, es necesario que esas leyes mayores o leyes de origen estén grabadas en la naturaleza. Es la naturaleza la que exhibe la voluntad del legislador supremo. De ahí que se pueda hacer un paralelo entre lo que entienden los pobladores del Tayrona con los misak en relación al origen y la naturaleza de las leyes mayores. Las palabras de Kwin Chaparro son precisas para explicar este punto:

\footnotetext{
21 Véase Joana Peña, supra, nota 7. Pág. 112

22 Id.

23 Id.
} 
Derecho Mayor, porque el creador es mayor, es la Ley de Origen, creó el mundo y dejó con la ley, dejó con la ley mayor. La ley de origen no la inventamos, porque muchos inventan, miles inventan, nosotros no inventamos sino ahí estamos como origen, siempre queremos estar como origen ${ }^{24}$.

El Derecho mayor es un derecho heredado que no ha sido creado por los hombres sino por la divinidad. Es el derecho que se encuentra grabado en los ritos de las comunidades y que busca la preservación de la tierra y la fertilidad de la comunidad. Sin embargo, el Estado colombiano - según el sentimiento de muchos miembros de la comunidad-, no ha facilitado los procesos para que el Derecho mayor sea reconocido, violentando de esta manera su forma de percibir el mundo y de existir o actuar en él. Según el gobernador del Cabildo de Guambía: "El derecho mayor y el Derecho Propio, ha sido un tema que en su momento reprimido por el Estado, reprimió oficialmente cuando la toma de tierras por los Misak"25.

El Derecho mayor, al concebirse como un derecho divino, sin mácula, sin vicio, se convierte en una consigna vital para quienes regulan sus existencias bajo sus preceptos. Como se trata de un mandato del creador, el derecho a la tierra, a la fertilidad, a la prosperidad, se transforma en una misión de vida, una misión comunitaria, que no acepta tregua. De ahí que las comunidades indígenas de la zona hayan emprendido una campaña por el rescate de este derecho divino.

Es una misión de vida, de una vida concebida dentro de la cosmología de los misak. Una vida que solo se concreta cuando quien la vive está actuando. No se trata de una vida reflexiva a la manera de los estoicos, se trata de una vida que exige movimiento, actuación. Las palabras del Tata Floro Tunubala ilustran, de manera vehemente, la idea de una misión comunitaria de vida:

El Derecho Mayor, es un Derecho que genera pensamiento, que no es un pensamiento de estar mirando únicamente la historia, sino que el Derecho Mayor es fortalecido por la historia para que la gente en cualquier tiempo podamos tener, aplicar y exigir a través del Derecho Mayor ${ }^{26}$.

24 Mamo Kwin Chaparro. Primer Encuentro de Autoridades Ancestrales sobre Derecho Mayor-Misak Universidad, Valle de Wuampia. Abril 20 del 2015.

25 Tata Floro Tunubala. supra, nota 6.

26 Id.
El Derecho mayor, entonces, se configura como un deber impuesto por la naturaleza. Es ella quien dicta cuáles son los actos considerados armónicos y cuáles desarmonizan la comunidad creando conflictos, enfermedad y esterilidad. El Derecho mayor muestra cómo debe ser el trato con la tierra, y por ende, con la mujer. Se trata de un derecho que armoniza, cumpliendo con el principio de correspondencia y complementariedad, la figura de la mujer con la inmanencia de la divinidad en la tierra. En palabras de un taita:

El Derecho Mayor es una concepción, una concepción en la cual el Deber y el Derecho Mayor nos es dado por esa naturaleza, es una forma de ordenar, son unos parámetros naturales, en la cual si el hombre no estuviera dentro de esa naturaleza ese Deber y ese Derecho Natural funcionaría con ese equilibrio con el cual lo vemos funcionando ${ }^{27}$.

La comprensión del Derecho mayor pasa por entender cómo la comunidad debe propender, más que a la protección de la tierra y de las mujeres, a permitir que la naturaleza siga su curso, no interrumpir lo que ya la divinidad hace de manera perfecta. En ese afán por prestar no solo los cuidados a la tierra y a la comunidad, los misak están interesados por mejorar las relaciones con las comunidades de cultura occidental. También se convierte en un deber para los miembros de la comunidad el crear armonía en torno a sus comunidades, de tal manera que no se vea afectado el orden. Por lo tanto, en palabras del gobernador del Cabildo de Guambía: "El Derecho Mayor permite recrear nuevas relaciones con el mundo occidental. Pero que el Derecho Mayor desde su nacimiento ha declarado públicamente y abiertamente de doble vía, de esa doble vía pues hay que seguir profundizando"28.

El Derecho mayor, como un derecho vinculado a la tierra, a la naturaleza, obliga a pensar en el respeto por las perspectivas de todos los pueblos que se relacionen con la comunidad misak. Como bien insiste el gobernador: "es pensar que somos las autoridades indígenas y que el mundo blanco también tiene sus autoridades y que podamos sentar en el marco del respeto para profundizar las políticas nuestras y hablar de las políticas que implementa el Estado"29.

Ahora bien, el Derecho mayor se configura dentro de la cosmología misak también como un

$\begin{array}{ll}27 & I d . \\ 28 & I d . \\ 29 & I d .\end{array}$


monumento a la unión de los seres vivos. El Derecho mayor convoca a los miembros de la comunidad a recordar la importancia de la unión fraternal con todos los seres vivos. De ahí que algunos ornamentos de los rituales misak simbolicen ese llamado natural y divino a la unidad. El rector de la Misak Universidad explica la relación entre los ornamentos rituales y la exhortación de la divinidad a la búsqueda de la unidad y la armonía:

Nunachak significa fogón grande, y que en torno al fogón grande los Misak hemos organizado especie de una confederación Misak y que en esa confederación, en torno a ese fogón pues llegamos todos los Tatas Misak que gobiernan y que viven en otros municipios en otros departamentos en Colombia ${ }^{30}$.

En ese orden de ideas, el Derecho mayor es unidad y armonía entre todo lo que compone el cosmos, el universo misak. Adicional a esto, el Derecho mayor es un conocimiento, es un tipo de arte o ciencia que se aprende y que es trasmitida por la tradición oral. Es un Derecho heredado que se refleja en el pensamiento y en la naturaleza: es una herencia presente en lo metafísico y lo físico. En palabras de Floro Tunubala: "El Derecho Mayor, es un derecho y es una ciencia que nos dejaron nuestros ancestros, para las generaciones, y le digo una ciencia porque hay todo un pensamiento político jurídico"31.

El derecho mayor en la cosmología misak también es comprendido como palabra, pero no se trata de la palabra comprendida como entidad metafísica, sino como una acción. El misak cuando habla está actuando, está haciendo, está existiendo. Cuando la divinidad habla, cuando la naturaleza habla, está manifestando su ley, una ley que no admite contravención o reforma. El Derecho mayor se funda en la Palabra Mayor de la naturaleza. El taita Rodrigo Morales Tunubala expresó estas ideas de una manera más clara y ajustada a la cosmología misak:

El Derecho Mayor es una Palabra Mayor, es una Palabra Mayor que ordena y por ejemplo, la lluvia a su debido tiempo, el día a su debido tiempo, la noche a su debido tiempo, igualmente dentro del Derecho Mayor están los ciclos ${ }^{32}$.

Es decir, la Palabra Mayor constituye la expresión del Derecho mayor, y los hombres pueden escudriñar

\footnotetext{
$30 \quad$ Id.

31 Id.

32 Tata Floro Tunubala. supra, nota 6.
}

este Derecho mayor por medio de la observación de la naturaleza. El orden que expresa la naturaleza y la armonía de sus relaciones de complementariedad y correspondencia son una expresión de la divinidad. Ahora bien, esta Palabra Mayor, al ser cosmológica, tiene una cualidad especial en el mundo de los misak: la Palabra Mayor produce leyes independientes de la presencia de los hombres. El taita Rodrigo Morales Tunubala señala esta cualidad o característica de la forma que sigue: "Son leyes que están ordenadas naturalmente, no requieren de intervención o legislación del hombre ${ }^{33 "}$

\section{El DeRECHO MAYOR EN LA LEY MISAK Y EL MANIFIESTO del Pueblo Guambiano}

Si bien el Derecho mayor se encuentra asociado en la cultura misak como un elemento cosmológico y no requiere justificación por parte de los hombres, los misak han establecido, de manera escrita, una serie de manifestaciones legales que pueden entenderse como un pacto de la comunidad, vinculado profundamente con el recorrido histórico y con las deudas históricas que ha de cobrar la comunidad, el cual establece cómo deben darse las relaciones armónicas al interior y al exterior de la comunidad.

Al tratarse de un derecho relacionado con la tierra, los misak desarrollan, tanto en el manifiesto del pueblo como en la ley, una serie de normas relativas al trato con la tierra. Una de ellas reza como sigue: “Todas las tierras del territorio Misak serán destinadas prioritariamente a suplir los requerimientos del ciclo de vida e identidad Misak, en concordancia con nuestros Planes de Vida" ${ }^{34}$. Es decir, las tierras que fueron heredadas por sus ancestros, las cuales dentro de la cosmología misak se configuran como parte del creador mismo, deben tener como finalidad única, como teleología suprema, la destinación de los recursos que esta produzca al sustento no solo de los miembros de la comunidad, sino también las manifestaciones culturales. Esto demuestra una doble función de la tierra que fue explicada al inicio del presente artículo: la tierra representa el sustento de los hombres, al tiempo en que se comprende como la manifestación de la divinidad, o mejor, como la divinidad misma.

33 Tata Floro Tunubala. supra, nota 6.

34 Véase Proclama de la ley del Derecho Mayor. 
En la Ley Misak, los territorios, o mejor:

Las tierras que son aptas para producción deberán estar dedicadas en primer lugar a incrementar y mejorar la producción de alimentos saludables para el autoconsumo, con el fin de mejorar la nutrición, la salud y, en general, el bienestar de los misak. Los cultivos comerciales e industriales no podrán desplazar la producción de nuestros alimentos ${ }^{35}$.

Lo anterior indica como la prioridad que debe darse a las tierras es la de la manutención de los miembros de la comunidad, lo cual excluye de un lugar privilegiado las demás funciones de la tierra: la función simbólica (relacionada con los ritos y con la divinidad) y la función comercial. Cabe anotar aquí que, para los misak, es clara la cuestión sobre la imposición de prácticas y maneras de explotar la tierra por parte de Occidente, de ahí que en la ley misak se trate el tema de manera puntual y sin eufemismos:

La imposición de conceptos y sistemas de área crítica para la biodiversidad, desarrollo sostenible, canje de deuda por naturaleza, servicios ambientales, cadenas productivas, reservas estratégicas, y otros, así como la adopción de leyes y políticas de aguas, páramos, forestales, de desarrollo rural, etc. ${ }^{36}$.

Esto es considerado por los misak una ofensa a su milenario comportamiento frente a la naturaleza. incluso, afirma la Ley Misak:

Están llevando a la mercantilización de la naturaleza, subordinándola al lenguaje del capital, legitimando y delineando así una transformación jurídica y material que busca la instauración de la propiedad privada de la vida, lo cual afecta gravemente los derechos humanos fundamentales de dignidad e identidad de nuestros pueblos ${ }^{37}$.

Ahora bien, no solo se trata de abastecer la comunidad con alimentos y otras necesidades de orden fisiológico, sino que se trata de una norma que exige el mejoramiento continuo del producto que emana de la tierra, de la madre tierra. El producto extraído de la tierra no solo debe estar en óptima calidad para el consumo sino que, quizá en razón al contacto de las comunidades misak con las comunidades occidentales, estos alimentos deben ser nutritivos y

\footnotetext{
35 Id.

36 Véase Ley Misak.

$37 \quad I d$.
}

saludables. Estas nociones pueden indicar la penetración de la conciencia occidental en las comunidades indígenas: lo nutritivo y saludable son conceptos que no encuentran traducción en la mentalidad, o mejor, en la cosmología misak, toda vez que dentro de su concepción del universo la tierra provee lo bueno cuando se encuentra en armonía. Cabe desatacar que la legislación misak, como se describirá más adelante, ha adquirido conciencia de que la interacción con el hombre moderno, además de ser obligatoria, es necesaria para la conservación de la madre naturaleza, de la tierra y, por ende, del Derecho mayor.

Es claro que la legislación misak contempla la relación con el hombre moderno y con otras comunidades indígenas como una interacción necesaria en pro de la armonía del universo y de la supervivencia de la comunidad (asunto muy vinculado con la cosmología y con la equiparación de la mujer con la tierra). Las palabras consignadas en la proclama de la Ley Misak arrojan un poco de luz sobre la cuestión de la armonía necesaria para las buenas relaciones entre los misak y las demás comunidades, indígena o no. Incluso, al ser la Ley Misak una ley construida en torno a la tierra y, por tanto, al Derecho mayor, fue necesario que dicha normatividad contuviera una descripción específica de la forma en que se debe cohabitar con miembros de comunidades externas: "Los pobladores de los cascos urbanos que cohabitan con los Misak deben contribuir a proteger y conservar todo el patrimonio natural del municipio, entendiendo que éste es también para su beneficio" 38 . Con estas palabras la proclama de la ley del Derecho mayor quiere indicar que la relación con las comunidades aledañas a las tierras de la comunidad misak debe caracterizarse por permitir una comunicación en doble vía. Dicha comunicación debe propender a la protección y la conservación de la naturaleza, ya que esta es considerada un patrimonio (una divinidad, podría agregarse). El respeto por la tierra, en el Derecho mayor, es un concepto fundamental para los misak. Sin embargo, no es suficiente con que los miembros de la comunidad misak actúen y vivan conforme a esta consciencia, hace falta que las demás comunidades que interactúen con ella la adquieran también. Esto se debe a la cosmología presente en el derecho a la tierra, en el Derecho mayor que, como se afirmó, es una obligación divina y hace parte de un llamado trascendental. De ahí que el Derecho mayor contemple la necesidad de que las comunidades ajenas a la cosmología misak contribuyan, no solo con

38 Véase Proclama de la ley del Derecho Mayor, supra, nota 32 
el respeto, sino también con la protección y conservación del patrimonio natural.

En ese sentido, los misak comprenden, como queda estipulado en la proclama del Derecho mayor, que es necesario mantener relaciones cordiales y armoniosas con las comunidades aledañas y con los líderes de la cultura occidental: "Las autoridades Misak mantendrán relaciones permanentes con las autoridades nacionales para buscar solución a los problemas que nos aquejan"39.

Otro punto importante para comprender el Derecho mayor y su representación en la normatividad de la comunidad misak son las rencillas y las deudas ancestrales relacionadas con la tierra. Como bien se anotó, cuando se hizo referencia a la cosmología, los misak relacionan su territorio con la dualidad o, mejor, con la complementariedad visible entre lo masculino y lo femenino. Si hace falta uno de los componentes la tierra enferma, se desarmoniza. De ahí que las últimas manifestaciones de la comunidad al respecto estén relacionadas con la desarmonización que crea la falta de reconocimiento, por parte del Estado colombiano, de la parte territorial que representa la figura femenina en su cosmovisión.

Las deudas relacionadas con las tierras han motivado en los misak la necesidad de ordenar su cosmos, de recomponer, a partir del reclamo de las tierras usurpadas en las diferentes etapas históricas, la armonía perdida: "Porque al robar la tierra y el trabajo, también nos dividieron y separaron" ${ }^{40}$ He aquí la primera preocupación del pueblo misak frente a la pérdida de las tierras: la división, la desarmonización de los miembros de la comunidad por motivo de la des-ritualización de la tierra y del Derecho mayor. La separación del grupo, de los miembros de la comunidad, es considerada por lo misak — como ya se señaló con antelación- como un daño mayor para su cosmovisión. Parte de la validez de este argumento se evidencia en las palabras consignadas en el Manifiesto del Pueblo Guambiano:

Unos quedamos como miserables comuneros de nuestro antiguo resguardo devorado por los terratenientes, otros quedamos como terrajeros esclavizados al querer del patrón, y los que han tenido que irse lejos de la comunidad, como peones arrumados en los establos de las haciendas u obligados a comprar

39 Id

40 Primer Encuentro de Autoridades Ancestrales sobre Derecho Mayor-Misak Universidad. Valle de Wuampia. Abril 20 del 2015. tierra como si no tuviéramos derechos, casi todos trabajando separados por usureros y créditos $^{41}$.

Perder la tierra para los misak es perder la libertad. No se pude hablar de perder la vida en tanto los misak viven en la medida en que actúan. Sin embargo, perder la libertad y la identidad es una muestra de desarmonía, o mejor, es una consecuencia de la des-ritualización de la tierra. Al ser violentada la tierra, al ser maltratada y trabajada, no para los fines con los que la divinidad la otorgó, sino para fines comerciales, la comunidad se desintegra, pierde la cosmovisión y el sentido de unidad.

En ese sentido, y para resumir lo que significa el Derecho mayor, se pude decir que se trata de un llamado divino, hecho a los miembros de la comunidad misak, hacia la recuperación de la armonía, a la recuperación de la correspondencia y complementariedad presentes en la tierra (en la cosmovisión de esta) y, en general, en la creación. Es una consigna que no admite contrariedad ni espera, como bien lo señala el Manifiesto del Pueblo Guambiano: "Esto es nuestro Derecho Mayor. Por encima de todos nuestros enemigos" ${ }^{42}$ Es decir, no existe límite, ni intereses particulares que puedan refrenar la reclamación de las tierras, de la armonía del pueblo misak. Como se ha señalado con antelación, la consigna de la reclamación de la tierra, por medio del Derecho mayor, se hace pensando en condiciones armoniosas de comunicación entre las diversas culturas; sin embrago, al ser una petición divina y una obligación existencial, los misak muestran su disposición ultranza a la reclamación, a cualquier costo. Incluso, a pesar de que el Derecho mayor está fundado sobre una base cosmológica y armónica, la Ley Misak es clara al insistir que el Derecho mayor está por encima de cualquier tipo de orden jurídico: "Por encima de sus escrituras, Por encima de sus leyes, Por encima de sus armas, Por encima de su poder"43.

El Derecho mayor no es negociable, es un derecho heredado que se desprende de la tierra, del derecho que tienen quienes recibieron estas tierras de sus ancestros quienes "siempre" habitaron América. Por esta razón, el Derecho mayor puede entenderse, de manera resumida, por medio de las palabras que siguen:

\footnotetext{
41 Véase Manifiesto del Pueblo Guambiano. Numeral 9. Disponible en https://alakusreikya.files. wordpress.com/2013/10/ manifiesto-guambiano.pdf

42 Id.

43 Véase Ley Misak, supra, nota 34.
} 
Por el derecho mayor, Por derecho de ser primeros. Por derecho de ser auténticos americanos. En esta gran verdad nace todito nuestro derecho todita nuestra fuerza. Por eso debemos recordarla, transmitirla y defenderla ${ }^{44}$.

En conclusión, el Derecho mayor es el derecho que tienen los hijos de esta tierra a disfrutar de ella, no solo con fines fisiológicos, sino con fines cosmológicos y existenciales. Es el Derecho que se basa en la armonía, en los principios de correspondencia y complementariedad. Es el derecho a la promulgación y desarrollo, por medio de los ritos y costumbres, de la cultura misak. Es el derecho a la fertilidad, a la conservación de la comunidad. En suma, es un derecho innegociable.

\section{REFERENCIAS}

Joana Peña. En las voces del pishimisak: apuntes etnográficos sobre el tiempo misak. Maguaré 23. 2009. Págs. $343-$ 369.

Josef Estermann. FILOSOFÍA ANDINA: SABIDURÍA INDÍGENA PARA UN MUNDO NUEVo. Instituto Superior Ecuménico Andino de Teología. (2006).

Luis Vasco. Conceptos Básicos de la Cosmovisión Guambiana en relación con sus procesos de lucha. Relatoría. 1997.

Manifiesto del Pueblo Guambiano. Numeral 9. Disponible en https://alakusreikya.files.wordpress.com/2013/10/ manifiesto-guambiano.pdf 